\title{
Study of Opportunistic Pathogenic Microflora in Otodectosis of Small Domestic Animals
}

\author{
A.R. Mkrtchyan, H.Z. Naghashyan, O.V. Scherbakov \\ Armenian National Agrarian University \\ artur.veterinar@rambler.ru, naghov1950@rambler.ru, oleg1vet@yandex.ru
}

\section{A RT I CLE IN F O}

Keywords:

otitis,

domestic animal,

mite,

bacterium,

antibiotic

\begin{abstract}
A B S T R A C T
According to statistical data, $10-20 \%$ of dogs and cats get an appointment with a veterinarian because of otitis externa - inflammation of the skin of the external auditory canal. The large role in the development of the inflammatory process in the auditory canal belongs to the epidermal staphylococcus, which often complicates the course of otitis caused by the parasitic ear mite Otodectes cynotis. The high sensitivity of epidermal staphylococcus to ampicillin, cefazolin and levomycetin allows us to recommend these antibiotics as a means of local treatment along with acaricides and antihistamines in the complex therapy of otodectosis of small domestic animals.
\end{abstract}

\section{Introduction}

Inflammation of the skin of external, middle and internal auditory canal or otitis media is a fairly common disease of small domestic animals, the causes of which can be mechanical damage, ear mite Otodectes cynotis, pathogenic fungi Malassezia pachydermatis, allergic reactions and autoimmune diseases. Thus, otitis media can act as both independent and secondary diseases (Belov and Danilov, 1990). According to statistics, 10-20\% of dogs and cats get an appointment with a veterinarian because of otitis externa - inflammation of the skin of the external auditory canal. Despite the variety of causes, the clinical picture of otitis media is very specific, and hence, the treatment of animals based only on clinical diagnosis is ineffective. Animals with otitis media are characterized by a head tilted towards the affected ear, head shaking, scratching in the area of the external auditory canal due to itching, unpleasant smell, and sometimes a leakage from the auditory canal, as well as painful reaction and fluctuation during external palpation of the auricle root (https://www. svoydoctor.ru, 2018). Otitis media is dangerous with its complications, since the inflammatory process that spreads along the continuation of the tissues with otitis media can lead to the development of inflammation of the membranes of the brain and cause death or permanent disability of the animal. From the point of view of complications, the greatest danger is otitis media of the inner ear - labyrinthitis, in case of which not only hearing loss occurs due to damage to the eardrum, but also there is a direct risk of developing meningitis and encephalitis. 
A large role in the development of the inflammatory process in the auditory canal belongs to opportunistic pathogenic bacteria, which can be both the main cause of otitis media, for example, with mechanical damage to the skin or the ingress of water and foreign bodies into the auditory canal, and a factor that complicates the inflammatory process caused by a parasitic ear mite, yeast fungi or an allergic reaction of the body. In the diagnosis of otitis media, it is necessary to identify not only the primary and secondary causes of the disease (mites, allergic reactions, yeast fungi and bacteria), but also predisposing and supporting factors that increase the risk of the disease (abundance of hair and maceration of the skin in the external auditory canal, hypersecretion of earwax and epithelial hyperkeratosis). Only a comprehensive approach to the diagnosis of otitis media, including clinical and instrumental, parasitological and bacteriological methods of research, can become the key to therapy and prevention.

In our research, we set out to determine the specific composition of the microflora in ear scabies (Otodectosis) of dogs and cats and its sensitivity to the most commonly used antibiotics in veterinary practice. The results of the research will help to identify the main culprit of bacterial complications in parasitic otitis media of small domestic animals and suggest the most effective drug for local therapy.

\section{Materials and methods}

The research was conducted in the laboratory of the Department of Epizootology and Parasitology of the Armenian National Agrarian University. Four cats and four dogs were under study, who were diagnosed with otodectosis (ear scabies); the age of the examined animals of different breeds ranged from 6 to 24 months. The animals belonged to different guardians and were in different housing and feeding conditions. The diagnosis of otodectosis was made on the basis of microscopic examination of the skin scraping of the external auditory canal, in which the tick Otodectes cynotis from the family Psoroptidae was found. From the contents of the external auditory canal of patients with otodectosis seeding on differential diagnostic nutrient media was made, such as Bismuth-Sulfite Agar and Endo Medium for the isolation of Salmonella and Escherichia, respectively, as well as on a selective nutrient medium - Mannitol Salt Agar for the isolation of Staphylococci. After seeding, the culture media were placed in a thermostat at $37{ }^{\circ} \mathrm{C}$ for 24 hours. The sensitivity of microbial colonies obtained in the culture medium to antibiotics was determined by the disc diffusion method (Kuzmin and Sefershaev, 1959). Bacterial smears were prepared by the conventional method on glass slides and after fixing over the flame of the burner, they were colored by Gram. Microscopic examination of the smears was performed under the immersion system of the microscope using the lens 90 and the eyepiece 15 . The total number of bacteria in the studied samples (content of the external auditory canal) was determined by direct counting method in fixed and colored bacteriological preparations according to Burgwitz methodology (Afanaseva, 1976). The number of microorganisms in $1 \mathrm{~g}$ of the test sample was determined by the formula:

$$
N=n P Q / p q g,
$$

where $n$ is the arithmetic mean number of cells in one field of view, $P$ - area of the preparation $\left(400 \mathrm{~mm}^{2}\right)$, $p$ - area of the microscope field of view, $\mathrm{mm}^{2}, Q-$ the amount of water to dilute the sample (500 or $250 \mathrm{ml}$ ), $q$ - volume of one drop of suspension (ml), $g$ - the amount of pathological material taken.

\section{Results and discussions}

Clinical and instrumental examination of the experimental animals indicated that different degrees of pain and fluctuation is observed when palpating the root of the affected ear; almost all animals had itching and scratching on the outer surface of the auricle, and black - brown accumulations of purulent exudate mixed with earwax were found in the external auditory canal. The body temperature of the examined animals remained within the upper norm limits during the entire treatment period.

Bacteriological examination of the contents of the external auditory canal revealed the following results: on bismuth sulfite agar and on the endo medium, single white colonies with a grayish tinge, smooth surface and even edges were observed, and on mannitol - salt agar, a continuous growth of colonies of white with a grayish tinge of mucosal plaque occupying the entire surface of the nutrient medium was found.

In smears prepared from colonies grown on the listed nutrient media, gram-positive cocci were found, located in the field of view of the microscope in clusters resembling bunches of grapes. The cultural, morphological and tinctorial features of the detected microorganisms allow us to identify them as white epidermal staphylococcus (staphylococcus epidermidis), which is often found on the skin and mucous membranes of humans and animals and can provoke purulent wound infections (Larry, et al., 2019). The average number of detected microorganisms per gram content of the external auditory canal in dogs 
turned out to be more than in cats, which is explained by the highest species resistance of the cat body to oppoertunistic pathogenic microflora (Table 1).

Table 1. Results of bacteriological examination of the contents of the external auditory canal in animals with otodectosis*

\begin{tabular}{|c|c|c|c|}
\hline $\begin{array}{c}\text { Average number of } \\
\text { staphylococci in the field } \\
\text { of view of the microscope }\end{array}$ & $\begin{array}{c}\text { Average number of } \\
\text { staphylococci per gram content } \\
\text { of the external auditory canal }\end{array}$ \\
\hline In dogs & In cats & In dogs & In cats \\
\hline 80 & 60 & 47059 & 35295 \\
\hline
\end{tabular}

Table 2. Sensitivity of staphylococcal strains isolated from animals with otodectosis to antibiotics*

\begin{tabular}{|l|c|c|}
\hline \multirow{2}{*}{ Name of the antibiotic } & \multicolumn{2}{|c|}{ Growth retardation zone (mm) } \\
\hline In dogs & In cats \\
\hline Ampicillin & 2.9 & 2.8 \\
\hline Tetracycline & 0.2 & 0.3 \\
\hline Streptomycin & 1.0 & 1.2 \\
\hline Cefazolin & 2.5 & 2.7 \\
\hline Levomycetin & 2.3 & 2.4 \\
\hline * Composed by the authors. & & \\
\hline
\end{tabular}

At the same time, when determining the sensitivity of the isolated strains of Staphylococcus to antibiotics by the disc diffusion method, it was revealed that all of them, regardless of the species of the animal from which they were isolated, are most sensitive to ampicillin, cefazolin and levomycetin (Table 2). When determining the antibiotic resistance of the isolated strains, the choice fell on a limited number of antibiotics that are widely used in practical veterinary medicine, are easily available and relevant for use.

The results of the studies are consistent with the literature data indicating the leading role of epidermal staphylococcus in the exacerbation of various purulent - inflammatory processes and its high sensitivity to antibiotic therapy.

\section{Conclusion}

Based on the results of the conducted research, the following conclusions can be drawn:

1. The main factor complicating the inflammatory process in otodectosis of small domestic animals is epidermal staphylococcus;

2. The average number of staphylococci in the contents of the external auditory canal in dogs exceeds the same indicator in cats, which indicates a relative species difference in the general nonspecific resistance to conditionally pathogenic microflora in these animal species;

3. The sensitivity to antibiotics in different strains of epidermal staphylococcus isolated from otodectosis patients in dogs and cats does not correlate with the age and type of animals.

4. The high sensitivity of epidermal staphylococcus to ampicillin, cefazolin and levomycetin allows us to recommend these antibiotics along with acaricides and antihistamines in the form of drops and ointments in the complex therapy of otodectosis of small domestic animals.

\section{References}

1. Afanaseva, O.V. (1976). Microbiological Control of Bakery Production. M., - pp. 65-67.

2. Belov, A.D., Danilov, Ye.P. (1990). Diseases of Dogs. M., -368 p.

3. https://www.svoydoctor.ru External Otitis of Dogs, 20 February 2018 (accessed on 03.09.2020).

4. Kuzmin, V.V., Sefershaev, M.A. (1959). Workshop on Veterinary Microbiology. M., - pp. 110-113.

5. Larry, M. Bush, MD, FACP. Charles E. Schmidt College of Medicine, Florida Atlantic University. Maria T. Vazquez-Pertejo. MD, FACP. Wellington Regional Medical Center, Staphylococcal Infection. June 2019. http://www.msdmanuals.com (accessed on 01.10.2019). 